Hautarzt 2022 $\cdot 73: 641-646$ https://doi.org/10.1007/s00105-021-04933-y Angenommen: 8. Dezember 2021 Online publiziert: 5 . Januar 2022

(c) Der/die Autor(en) 2022

\section{Ungewöhnliche Präsentation einer Psoriasis vulgaris bei einem 11-jährigen Patienten}

Viktoria Gruber · Wolfgang Weger · Lorenzo Cerroni · Barbara Binder

Universitätsklinik für Dermatologie und Venerologie, Medizinische Universität Graz, Graz, Österreich

\title{
Zusammenfassung
}

Die Psoriasis vulgaris tritt mit einer Prävalenz von bis zu $2 \%$ im Kindes- und Jugendalter auf. Größtenteils wird die Diagnose klinisch gestellt. Wir berichten über einen pädiatrischen Patienten, welcher uns aufgrund der für eine Psoriasis vulgaris eher untypischen Lokalisation vor eine diagnostische Herausforderung stellte. Diskutiert werden die wichtigsten Differenzialdiagnosen der verschiedenen Psoriasisformen sowie die aktuellen Therapieempfehlungen im Kindes- und Jugendalter.

\section{Schlüsselwörter}

Pädiatrie · Psoriasis vulgaris · Klinische Varianten · Differenzialdiagnosen · Therapieoptionen

\section{Anamnese}

Ein 11-jähriger Patient stellte sich in Begleitung seiner Mutter in der Ambulanz für pädiatrische Dermatologie aufgrund juckender therapieresistenter Hautveränderungen am Gesäß seit 4 Jahren vor. Die bisherigen lokalen Behandlungen umfassten blande Externa, Antimykotika, Glukokortikoide sowie Antibiotika. Der Patient nahm keine Dauermedikation ein. In der Krankengeschichte fanden sich neben einer Phimose im Kleinkindalter und einer Schädelprellung nach einem Fahrradsturz keine Besonderheiten. In der Familienanamnese

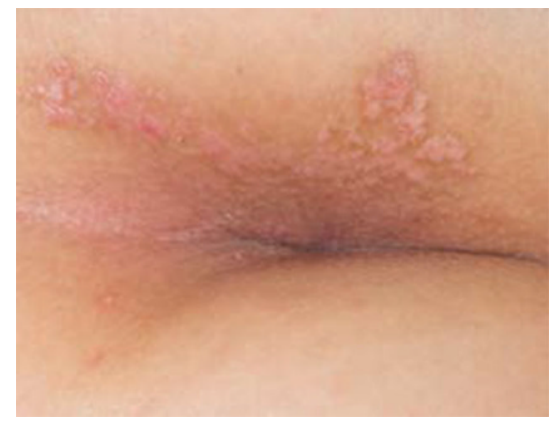

Abb. 1 \ Lokalbefund an der Gesäßfalte mit juckenden, teils konfluierenden Plaques. Eine Schuppung ist kaum vorhanden war zu erheben, dass sowohl ein Onkel als auch eine Cousine des Jungen an einer Psoriasis vulgaris litten.

\section{Klinischer Befund}

In der dermatologischen Untersuchung zeigten sich in der Gesäßfalte und der rechtsseitigen Glutealregion mehrere hautfarbene bis blassrote, teils konfluierende kleinpapulöse Plaques mit teilweise nur diskreter weißlicher Schuppung und stellenweise oberflächlichen Exkoriationen (• Abb. 1).

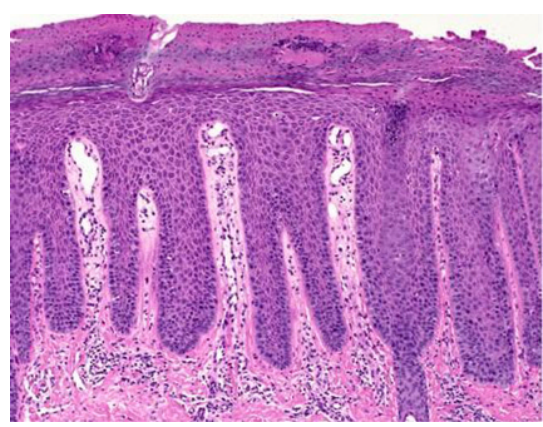

Abb. $2 \Delta$ Der histopathologische Befund ergab eine psoriasiforme Epidermishyperplasie mit Parakeratose und Verlust des Stratum granulosum. Auffallend sind auch verlängerte dermale Papillae mit teleangiektatisch erweiterten Kapillaren 


\section{Kasuistiken}

Tab. 1 Wichtige Differenzialdiagnosen der Psoriasis und deren klinische Unterscheidungsmerkmale. (Mod. nach Blume-Peytavi et al. [2]) Psoriasisform $\quad$ Differenzialdiagnosen mit Unterscheidungsmerkmale

Psoriasis vulga- $\quad$ Seborrhoisches Ekzem

ris (nummularis) oberer Stamm, Intertrigines)

Fehlen von Nagelveränderungen

Atopisches Ekzem

Unscharf begrenzte erythematöse Plaques und exkoriierte Papeln

Juckreiz meist stärker als bei der Psoriasis ausgeprägt

Aussparung der Windelregion, Assoziation mit Allergien und Asthma bronchiale

Nummuläres Ekzem

Relativ scharf begrenzte, runde erythematöse Plaques mit Schuppung oder feinen Fissuren zwischen 1 und $10 \mathrm{~cm}$ Durchmesser an Stamm und Extremitäten

Aussparung von Gesicht und Kopfhaut

Erythrokeratodermia symmetrica progressiva

Scharf begrenzte erythematöse hyperkeratotische Plaques an Streckseiten der Extremitäten

Psoriasis guttata (exanthematica)

\section{Pityriasis rosea}

Akutes Auftreten multipler erythematöser Papeln und Plaques mit Collerette-Schuppung an Stamm und proximalen Extremitäten, häufig Beginn mit „Primärmedaillon"

Meist Aussparung des Gesichts und der Intertrigines

Tinea corporis

Scharf begrenzte rundovale Erytheme oder Plaques mit randbetonter Schuppung oder pustulösem Randsaum und zentralem

Auslöschphänomen

Erregernachweis kulturell

Pityriasis lichenoides chronica

Chronisch rezidivierender Verlauf mit Auftreten disseminierter erythematöser Papeln mit zentraler Deckelschuppe an Stamm und Extremitäten

Aussparung des Kopfes

Psoriasis inversa Intertriginöse Kandidose

Erytheme mit abstreifbaren weißen Belägen, im Randbereich papulopustulöse Satelliten

Erregernachweis mikroskopisch, kulturell

Irritative Windeldermatitis

Scharf begrenzte Rötungen und erythematöse Papeln mit Erosionen oder Mazeration an Gesäß, Genitale, Unterbauch und Oberschenkeln

Aussparung der Hautfalten

Erythro-

dermatische Kutane T-Zell-Lymphome

Psoriasis

Erythrodermie und Schuppung mit Lymphadenopathie

Diagnosesicherung histologisch, immunhistologisch

Morbus Leiner

Maximalvariante des seborrhoischen Säuglingsekzems mit Erythrodermie und fettig-lamellöser Schuppung

Diarrhö, Infektneigung und Gedeihstörungen

Kongenitale ichthyosiforme Erythrodermie

Erythrodermie mit heller feiner Schuppung („Kollodiumbaby“)

Gestörte Temperaturregulation mit Neigung zu Hyperthermie

Psoriasis pal-

Tinea manuum et pedum

moplantaris

Palmoplantare Erytheme, Schuppen mit teilweise Bläschen oder Rhagaden

Erregernachweis kulturell

Diagnose

Zur weiterführenden Diagnostik wurde eine Probebiopsie (4-mm-Stanze) in Lokalanästhesie entnommen (• Abb. 2).
Therapie und Verlauf

Unser Patient hat eine kurzfristige Lokaltherapie mit einem Glukokortikoid der Klasse III erhalten, welches im Anschluss durch ein topisches Vitamin-D-Derivat ersetzt wurde. Vier Wochen nach Einleitung der Therapie war der störende Juckreiz bereits abgeklungen, und die Hautveränderungen sind flacher geworden. Im Rahmen der Langzeitbetreuung 


\begin{tabular}{|l|l|}
\hline Tab. 1 (Fortsetzung) \\
\hline Psoriasisform & Differenzialdiagnosen mit Unterscheidungsmerkmalen \\
\hline Psoriasis capitis & Seborrhoisches Säuglingsekzem \\
\cline { 2 - 3 } & $\begin{array}{l}\text { Gelblich-fettige Schuppung an der Kopfhaut (frontoparietal) mit mildem Juckreiz, wobei die Stirn-/Nackenhaargrenze nicht über- } \\
\text { schritten wird }\end{array}$ \\
\cline { 2 - 3 } & Häufig Beginn im Gesicht mit erythematösen schuppenden Plaques \\
\cline { 2 - 3 } & Tinea capitis (Mikrosporie) \\
\cline { 2 - 3 } & Meist juckende Alopezieherde mit feinlamellärer Schuppung und Erythem \\
\cline { 2 - 3 } & Erregernachweis mikroskopisch, kulturell \\
\cline { 2 - 3 } & Pityriasis (Tinea) amiantacea \\
\cline { 2 - 3 } & Festhaftende asbestartige Schuppung an Haaren und Kopfhaut \\
\cline { 2 - 3 } & Atopisches Kopfhautekzem \\
\cline { 2 - 3 } & Erytheme, Papulovesikel und Krusten („Milchschorf“) in der Regel ab dem 3. Lebensmonat \\
\hline
\end{tabular}

\begin{tabular}{|c|c|c|}
\hline \multicolumn{3}{|c|}{ Spezifische topische Therapien } \\
\hline $\begin{array}{l}\quad \text { "wird empfohlen“ } \\
\text { Glukokortikoide } \\
\text { - Klasse II (in empfindlichen Arealen) } \\
\text { - Klasse III } \\
\text { Vitamin-D-Derivate } \\
\text { - Calcipotriol ab } 6 \text { Jahren } \\
\text { - Tacalcitol ab } 12 \text { Jahren } \\
\text { Dithranol (stationär) ab } 1 \text { Jahr }\end{array}$ & $\begin{array}{l}\text { „'kann empfohlen werden“ } \\
\text { Calcineurininhibitoren” (in } \\
\text { empfindlichen Arealen) } \\
\text { - Pimecrolimus ab } 2 \text { Jahren } \\
\text { - Tacrolimus ab } 2 \text { Jahren }\end{array}$ & $\begin{array}{l}\text { „kann erwogen werden }{ }^{{ }^{\prime \prime}} \\
\text { Tazaroten } \\
\text { Dithranol (ambulant) ab } 1 \mathrm{Jahr}\end{array}$ \\
\hline \multicolumn{3}{|c|}{ Systemische Therapien } \\
\hline $\begin{array}{l}\qquad \text { "wird empfohlen“ } \\
\text { Acitretin }{ }^{*} \text { bei Mädchen und Jungen } \\
\text { vor der Pubertät und bei männlichen } \\
\text { Jugendlichen bei pustulösen Formen } \\
\text { Methotrexat } \\
\text { Adalimumab ab } 4 \text { Jahren } \\
\text { Bei nicht ausreichendem } \\
\text { Therapieerfolg, Unverträglichkeit, } \\
\text { Kontraindikation von Adalimumab } \\
\text { oder MTX: } \\
\text { Etanercept ab } 6 \text { Jahren } \\
\text { Ustekinumab ab } 12 \text { Jahren }\end{array}$ & $\begin{array}{l}\text { „kann empfohlen werden }{ }^{2 \prime \prime} \\
\text { Ciclosporin als Induktionstherapie } \\
\text { Fumarsäureester }\end{array}$ & $\begin{array}{l}\quad \text { „kann erwogen werden }{ }^{3 \text { “ }} \\
\text { Apremilast } \\
\text { Brodalumab } \\
\text { Certolizumab pegol } \\
\text { Guselkumab } \\
\text { Infliximab } \\
\text { Ixekizumab ab } 6 \text { Jahren } \\
\text { Secukinumab ab } 6 \text { Jahren }\end{array}$ \\
\hline \multicolumn{3}{|c|}{$\begin{array}{l}\text { Wirkstoff- und duftstofffreie Externa +/-Zusatz von Urea, Glycerin, Polidocanol 3-10\% } \\
\text { ggf. Keratolytika }\end{array}$} \\
\hline \multicolumn{3}{|c|}{ ggf. begleitende psychosoziale Therapie } \\
\hline & $\begin{array}{l}\text { UV-B-Schmalspektrum } \\
\text { In Einzelfällen bei Adoleszenten }\end{array}$ & \\
\hline
\end{tabular}

Abb. $3<$ Therapieoptionen der Psoriasis vulgaris im Kindes- und Jugendalter. ${ }^{1}$ Bei nicht ausreichendem Therapieerfolg, Unverträglichkeit, Kontraindikation gegenüber anderen topischen Therapieoptionen. ${ }^{2}$ Bei nicht ausreichendem Therapieerfolg, Unverträglichkeit, Kontraindikation von Adalimumab oder MTX. ${ }^{3}$ Bei nicht ausreichendem Therapieerfolg,Unverträglichkeit, Kontraindikation anderer systemischer Therapieoptionen. Asterisk Fehlende Zulassung für die Indikation Psoriasis. (Mod. nach Eisert et al. [5]) werden an unserer Klinik Kontrollen in 3-monatigen Abständen durchgeführt.

\section{Diskussion}

Die Psoriasis vulgaris tritt sowohl im Kindes- als auch Erwachsenenalter in etwa
$70 \%$ der Fälle als Plaquepsoriasis auf [11]. Die Schuppung und Infiltration der Herde können jedoch bei Kindern milder als bei Erwachsenen ausgeprägt sein [3].

Etwa $30 \%$ der Kinder mit Psoriasis vulgaris präsentieren sich initial mit einer Psoriasis guttata. Diese akute Verlaufsform tritt häufig in Assoziation mit einer Infektion durch $\beta$-hämolysierende Streptokokken oder Viren auf. In diesem Zusammenhang zählen die Angina tonsillaris als auch die streptogene perianale Dermatitis zu den häufigsten Auslösern [11]. 
Seltenere Varianten beinhalten unter anderem die Psoriasis inversa, Windelpsoriasis, Psoriasis pustulosa, Psoriasis palmoplantaris und die Erythrodermie [7].

Eine Besonderheit bei pädiatrischen $\mathrm{Pa}$ tienten mit Psoriasis vulgaris stellt die bevorzugte Beteiligung bestimmter Hautareale wie etwa Gesicht, Capillitium, Intertrigines und Windelbereich dar. Im Gegensatz zur irritativen Windeldermatitis sind bei der Windelpsoriasis auch die Inguinalfalten mit betroffen. Eine Beteiligung von Nägeln oder Gelenken wird bei jeweils bis zu $40 \%$ der Betroffenen beobachtet [12].

In der Regel wird die Diagnose einer Psoriasis vulgaris anhand des typischen klinischen Erscheinungsbildes gestellt. Dennoch können seltenere Psoriasisvarianten und das Auftreten psoriatischer Hautveränderungen an atypischen anatomischen Lokalisationen eine diagnostische Herausforderung darstellen. Bei nicht eindeutigem klinischem Befund ist eine Probebiopsie zur histologischen Diagnosesicherung notwendig.

Die Differenzialdiagnosen umfassen unter anderem das seborrhoische und atopische Ekzem, die Pityriasis rosea, Epidermomykosen oder den Morbus Leiner (- Tab. 1).

Die Grundlage jeder Psoriasisbehandlung bildet die topische Therapie mit begleitender Basistherapie, welche in den meisten Fällen zum Erfolg führen. Eine zumindest 1-mal tägliche Anwendung wirkstofffreier Externa wird zum Schutz der Hautbarriere empfohlen. Je nach Ausmaß der Schuppung kann eine initiale Keratolyse angezeigt sein, um eine bessere Penetration der nachfolgend applizierten topischen Wirkstoffe gewährleisten zu können.

Topische Glukokortikoide der Klasse II und III werden als Wirkstoffe der ersten Wahl in der Initial- und Erhaltungstherapie der Psoriasis eingesetzt. Im Gesichts- und Genitalbereich sowie intertriginös sollten jedoch weniger potente Glukokortikoide für maximal 2 Wochen appliziert werden und im Anschluss durch topische Calcineurininhibitoren ersetzt werden [4]. Eine zunächst tägliche Applikation topischer Glukokortikoide mit langsamer Dosisreduktion und nachfolgender proaktiver Anwendung hat sich als zielführend erwiesen [1]. Kombinationsbehandlungen (Glukokorti- koide plus Vitamin-D-Derivate) kommen als Erstlinientherapie zum Einsatz [1, 10].

Bei schweren Verläufen ist eine Systemtherapie aufgrund unzureichender Wirksamkeit lokaltherapeutischer Maßnahmen notwendig - hier kommen diverse Immunsuppressiva, Biologika und orale Retinoide zum Einsatz.

Als langjährig eingesetztes Medikament bei Erwachsenen als auch pädiatrischen PatientInnen wird Methotrexat (MTX) als Erstlinientherapie der mittelschweren und schweren Plaquepsoriasis empfohlen [1]. Zusätzlich weist MTX ein günstiges Wirkungsprofil bei der Psoriasisarthritis, schweren Verläufen einer Psoriasis guttata sowie pustulösen und erythrodermatischen Verläufen auf [8]. Bei ausschließlich pustulösen Psoriasisformen wird eine Behandlung mit Acitretin, einem aromatischen Retinoid der zweiten Generation, empfohlen [8].

Zur Therapie der Psoriasis im Kindesund Adoleszentenalter sind die Biologika Adalimumab (ab dem 4. Lebensjahr) sowie Etanercept, Ustekinumab, Ixekizumab und Secukinumab (jeweils ab dem 6. Lebensjahr) zugelassenen $[6,9]$. Für weitere Biologika werden derzeit Phase-III-Studien zur Therapie der pädiatrischen Psoriasis durchgeführt [9].

Regelmäßiges Screening auf mögliche kardiovaskuläre, metabolische als auch psychiatrische Komorbiditäten sowie eine frühzeitige Intervention und begleitende psychosoziale Unterstützung runden den Therapieplan ab [6]. Eine Übersicht der Therapieoptionen im Kindes- und Jugendalter ist in - Abb. 3 angeführt.

\section{Fazit für die Praxis}

- Die Plaquepsoriasis bildet die häufigste Erscheinungsform der Psoriasis vulgaris im Kindes- und Jugendalter.

- Die Diagnose wird in der Regel klinisch gestellt. Seltene Psoriasisvarianten und das Auftreten psoriatischer Hautveränderungen an atypischen anatomischen Lokalisationen erfordern eine Probebiopsie.

- In den meisten Fällen sind lokaltherapeutische Maßnahmen für einen Therapieerfolg ausreichend.

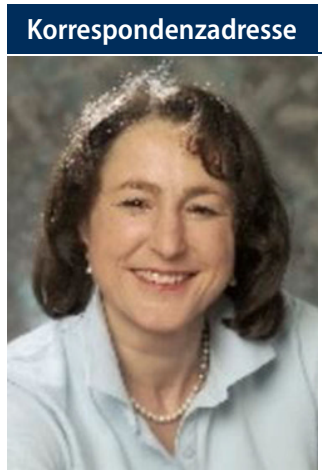

\section{PD Dr. med. univ. Barbara Binder}

Universitätsklinik für Dermatologie und Venerologie, Medizinische Universität Graz Auenbruggerplatz 8, $8036 \mathrm{Graz}$, Österreich barbara.binder@medunigraz.at

Funding. Open access funding provided by Medical University of Graz.

\section{Einhaltung ethischer Richtlinien}

Interessenkonflikt. V. Gruber, W. Weger, L. Cerroni und B. Binder geben an, dass kein Interessenkonflikt besteht.

Für diesen Beitrag wurden von den Autoren keine Studien an Menschen oder Tieren durchgeführt. Für die aufgeführten Studien gelten die jeweils dort angegebenen ethischen Richtlinien. Für Bildmaterial oder anderweitige Angaben innerhalb des Manuskripts, über die Patienten zu identifizieren sind, liegt von ihnen und/oder ihren gesetzlichen Vertretern eine schriftliche Einwilligung vor.

Open Access. Dieser Artikel wird unter der Creative Commons Namensnennung 4.0 International Lizenz veröffentlicht, welche die Nutzung, Vervielfältigung, Bearbeitung, Verbreitung und Wiedergabe in jeglichem Medium und Format erlaubt, sofern Sie den/die ursprünglichen Autor(en) und die Quelle ordnungsgemäß nennen, einen Link zur Creative Commons Lizenz beifügen und angeben, ob Änderungen vorgenommen wurden.

Die in diesem Artikel enthaltenen Bilder und sonstiges Drittmaterial unterliegen ebenfalls der genannten Creative Commons Lizenz, sofern sich aus der Abbildungslegende nichts anderes ergibt. Sofern das betreffende Material nicht unter der genannten Creative Commons Lizenz steht und die betreffende Handlung nicht nach gesetzlichen Vorschriften erlaubt ist, ist für die oben aufgeführten Weiterverwendungen des Materials die Einwilligung des jeweiligen Rechteinhabers einzuholen.

Weitere Details zur Lizenz entnehmen Sie bitte der Lizenzinformation auf http://creativecommons.org/ licenses/by/4.0/deed.de. 


\section{Literatur}

1. AWMF online (2018) S2k-Leitlinie Therapie derPsoriasis bei Kindern und Jugendlichen. https://www. awmf.org/uploads/tx_szleitlinien/013-094I_S2k_ Therapie-Psoriasis-Kinder-Jugendliche_2019-07. pdf.Zugegriffen:21.Sept. 2021

2. Blume-Peytavi U et al (2018) Atlas der pädiatrischen Dermatologie, S392-394

3. Bronckers IM, Paller AS, van Geel MJ, van de Kerkhof PC, Seyger MM (2015) Psoriasis in children and adolescents: diagnosis, management and comorbidities. Paediatr Drugs 17(5):373-384

4. Brune A, Miller DW, Lin P, Cotrim-Russi D, Paller AS (2007) Tacrolimusointment is effective forpsoriasis on the face and intertriginous areas in pediatric patients. Pediatr Dermatol 24(1):76-80

5. Eisert L et al (2019) S2k-Leitlinie zur Therapie der Psoriasis bei Kinder und Jugendlichen, $\mathrm{S} 864$

6. Mahé E (2020) Optimal management of plaque psoriasis in adolescents: current perspectives. Psoriasis (Auckl) 10:45-56

7. Morris A, Rogers M, Fischer G, Williams K (2001) Childhood psoriasis: a clinical review of 1262 cases. Pediatr Dermatol 18(3):188-198

8. Napolitano M, Megna M, Balato A, Ayala F, Lembo S, Villani A et al (2016) Systemic treatment of pediatric psoriasis: a review. Dermatol Ther (Heidelb) 6(2):125-142

9. Nogueira M, Paller AS, Torres T (2021) Targeted therapy for pediatric psoriasis. Paediatr Drugs 23(3):203-212. https://doi.org/10.1007/s40272021-00443-5

10. Oquendo M, Abramovits W, Morrell P (2012) Topical vitamin $D$ analogs available to treat psoriasis. SKINmed 10(6):356-360

11. Relvas M, Torres T (2017) Pediatric psoriasis. Am J Clin Dermatol 18(6):797-811

12. Tollefson MM (2014) Diagnosis and management of psoriasis in children. Pediatr Clin North Am 61(2):261-277

\section{Uncommon presentation of psoriasis vulgaris in an 11-year-old patient}

Psoriasis vulgaris occurs with a prevalence of up to $2 \%$ in childhood and adolescence. In most cases, diagnosis can be made clinically. We report about a pediatric patient with psoriasis vulgaris who posed a diagnostic challenge due to uncharacteristic manifestation. The most important differential diagnoses of various forms of psoriasis vulgaris as well as actual treatment recommendations in childhood and adolescence are discussed.

\section{Keywords}

Pediatrics · Psoriasis vulgaris · Clinical variants · Differential diagnosis · Treatment options 
Hier steht eine Anzeige.

黑 Springer 